\section{OC-072 OESOPHAGEAL CANCER - AN AETIOLOGICAL INVESTIGATION INTO THE POTENTIAL PROTECTIVE EFFECT OF STATINS IN THE UK GENERAL PRACTICE RESEARCH DATABASE (GPRD)}

doi:10.1136/gut.2011.239301.72

H Y Bhutta, ${ }^{1}$ A Clark, ${ }^{2}$ S Holt, ${ }^{3}$ M P N Lewis, ${ }^{1}$ A R Hart, $, 4,5,{ }^{*}$ Department of General Surgery, Norfolk and Norwich Hospital, UK; ${ }^{2}$ Department of Medical Statistics, University of East Anglia, UK; ${ }^{3}$ Roundwell Medical Centre, Norwich, UK; ${ }^{4}$ Department of Gastroenterology, Norfolk and Norwich Hospital, UK; ${ }^{5}$ School of Medicine, University of East Anglia, Norwich, UK

Introduction The incidence of oesophageal carcinoma has risen dramatically and is associated with a poor prognosis. Statins, primarily used for the prevention of cardiovascular disease, have anti-cancer properties in experimental work, although there is minimal epidemiological data in this area. The aim of this investigation was to address this deficiency by investigating if statins prevent the development of oesophageal carcinoma in an epidemiological prospective cohort study.

Methods The cohort consisted of approximately 4 million people registered in the General Practice Research Database (GPRD), a UK database of 488 nationwide general practices, which includes information on patients' medications prior to the development of illnesses, including cancer. Statin use was defined as a prescription for a minimum of 10 months preceding the diagnosis of oesophageal cancer. Each case was matched with four controls and conditional logistic regression used to estimate the odds ratios (ORs), plus 95\% confidence intervals (CIs), for the development of carcinoma. The ORs were adjusted for diabetes mellitus, body mass index, smoking, aspirin, NSAIDs, PPIs and drugs which relax the lower oesophageal sphincter.

Results A total of 4242 people developed oesophageal carcinoma (adenocarcinoma and squamous cancers combined) between 2000 and 2008 (65.3\% male, mean age 71.0 years, SD = 11.4 years) which were matched with 17233 controls. Statin use was negatively associated with the development of oesophageal carcinoma ( $\mathrm{OR}=0.84,95 \% \mathrm{CI}=0.73$ to 0.95 ). Categorisation into lipophilic and hydrophilic statins also demonstrated negative associations $(\mathrm{OR}=0.86,95 \% \mathrm{CI}=0.75$ to 0.98 and $\mathrm{OR}=$ $0.71,95 \% \mathrm{CI}=0.51$ to 0.98 , respectively). The median length of statin use in cases prior to diagnosis was 3.7 years (Interquartile range $=1.9-5.7$ years) and the magnitude of this negative association was similar for time periods extending beyond one year. The effect sizes of individual drugs could not be assessed due to the smaller numbers in these sub-groups.

Conclusion Regular use of statins may protect against the development of oesophageal carcinoma, although confirmation in other populations is required. The data may support an 
assessment of statins in randomised controlled clinical trials as chemopreventative measures in the general population and those with Barrett's oesophagus.

Competing interests None.

Keywords oesophageal cancer, statins. 